\title{
Milk fatty acids predict the foraging locations of the New Zealand fur seal: continental shelf versus oceanic waters
}

\author{
Alastair M. M. Baylis ${ }^{1,2,5 *}$, Peter D. Nichols ${ }^{3,4}$ \\ ${ }^{1}$ School of Earth and Environmental Sciences, University of Adelaide, Adelaide, South Australia 5005, Australia \\ ${ }^{2}$ South Australian Research and Development Institute (Aquatic Sciences), PO Box 120, Henley Beach, Adelaide \\ South Australia 5022, Australia \\ ${ }^{3}$ CSIRO Marine and Atmospheric Research, Food Futures Flagship, GPO Box 1538, Hobart, Tasmania 7001, Australia \\ ${ }^{4}$ Antarctic and Climate Ecosystems Cooperative Research Centre, University of Tasmania, Private Bag 80, \\ Tasmania 7001, Australia
}

${ }^{5}$ Present address: Falkland Islands Government, Fisheries Department, PO Box 598, Stanley, Falkland Islands

\begin{abstract}
Lactating New Zealand fur seals Arctocephalus forsteri utilise 2 ecological regions: continental shelf habitats and oceanic habitats associated with the Subtropical Front. Using milk fatty acids (FA) obtained from 29 satellite-tracked fur seals, we characterised the FA composition of seals that foraged on the continental shelf, and those that foraged in oceanic waters. Seals that foraged within oceanic waters were characterised by milk being comparatively high in monounsaturated fatty acids (MUFA; $47.4 \pm 4.4 \%$, mean \pm SD), and lower in polyunsaturated fatty acids $\left(\mathrm{PUFA}_{i} 23.8 \pm\right.$ $4.0 \%$ ) when compared to seals that foraged in continental shelf waters (MUFA $36.7 \pm 5.4$ and PUFA $31.4 \pm 5.5 \%$ ). Based on FA compositions, we predicted the likelihood that milk samples collected at random $(\mathrm{n}=131)$ represented individual seals having foraged either on the continental shelf or in distant oceanic waters. Results indicated that $74 \%(\mathrm{n}=97)$ of seals were likely to have foraged in oceanic waters, with $26 \%(\mathrm{n}=34)$ likely to have foraged within continental shelf waters. These results were supported by the small sub-sample of 29 satellite-tracked seals, which indicated that $62 \%$ of seals had foraged in oceanic waters. FA analysis and satellite-tracking results contrasted with scat analyses, from which only $7 \%$ of scats contained prey remains from oceanic waters. The results suggest scats were biased toward females foraging on the continental shelf. To further understand the diet of New Zealand fur seals, additional information on potential prey species that inhabit waters associated with the Subtropical Front south of Australia is required, as well as the continued development and application of alternative dietary techniques.
\end{abstract}

KEY WORDS: Milk fatty acids $\cdot$ New Zealand fur seal $\cdot$ Subtropical Front $\cdot$ Continental shelf

\section{INTRODUCTION}

Understanding ecosystem structure and function requires knowledge of trophic relationships between predators and their prey, and temporal and spatial aspects of predator and prey diets (Raclot et al. 1998). In wide-ranging marine predators, assessing predator-prey interactions over a spatial context is inherently difficult. Central-place foragers such as pro- cellariiform seabirds and pinnipeds often exploit multiple ecological regions (i.e. inshore waters and distant ocean fronts) over varying spatial scales (10s to 1000 s of kilometres) (Chaurand \& Weimerskirch 1994, Bradshaw et al. 2003, Beauplet et al. 2004). Generalizing the diet of marine predator populations by combining dietary information from individuals that forage in different ecological regions may disguise important trophic interactions (Paine 1988). To accurately repre- 
sent diet and to assess trophic interactions, the ability to separate dietary information from different ecological regions utilised by marine predators is required.

Traditionally the diets of marine predators have been largely inferred using scat, regurgitation and stomach samples. Although these methods have proven to be invaluable, it is often assumed they are only representative of the last foraging bout, and inherent biases associated with differences in the rates of passage of food through the gut and in the digestibility of particular prey are well established (Dellinger \& Trillmich 1988, Bodley et al. 1999, Staniland 2002). As such, the accuracy at which traditional methods are able to reconstruct the diet of wide-ranging marine predators is dependant, to some degree, on the characteristics of their foraging trips (such as distance and duration), which vary temporally (i.e. seasonal) and spatially (colony location) and between individuals (e.g. Beauplet et al. 2004, Gremillet et al. 2004, Robson et al. 2004, Staniland et al. 2007, Baylis et al. 2008a,b, 2009).

The New Zealand fur seal Arctocephalus forsteri is a wide-ranging marine predator and the most abundant seal species in the Australia/New Zealand region. Approximately $85 \%$ of Australia's population of New Zealand fur seals reside in the state of South Australia (Goldsworthy et al. 2003, Shaughnessy 2005). As a result of their abundance and size, New Zealand fur seals breeding in the state of South Australia are important consumers of pelagic resources within the southern Australian region (Goldsworthy et al. 2003). However, despite the importance of New Zealand fur seals as top predators, their increasing abundance and the potential for interaction with commercial fisheries, our understanding of the diet of New Zealand fur seals in South Australia is primarily derived from 1 study, conducted at 1 colony (Page et al. 2005) (limited dietary data are also presented in Goldsworthy et al. [2003]). These studies have indicated that lactating New Zealand fur seals predominantly foraged within continental shelf waters (Goldsworthy et al. 2003, Page et al. 2005, 2006).

Recent satellite-tracking studies at several New Zealand fur seal breeding colonies in South Australia have revealed that lactating New Zealand fur seals actually use 2 spatially discrete foraging habitats (Baylis et al. 2008a,b). These are continental shelf habitats (as recorded by Page et al. 2005, 2006) that are associated with a coastal upwelling and distant oceanic waters that are associated with the Subtropical Front (STF) (Baylis et al. 2008a,b). Seals that forage on the continental shelf travel shorter distances and have shorter foraging trip durations $(127 \pm 44 \mathrm{~km}$ and $8.1 \pm$ $4.5 \mathrm{~d}$, mean $\pm \mathrm{SD}$ ) when compared to seals that forage in oceanic waters, that travel up to $1000 \mathrm{~km}$ from the colony on foraging trips of up to $40 \mathrm{~d}$ duration (Baylis et al. 2008a,b). Given the vast distances that lactating New Zealand fur seals travel to forage, interpreting diet using traditional methods is unlikely to accurately represent the diet of seals foraging in distant oceanic waters, or provide a means by which to detect and quantify the spatial separation of foraging habitats.

Owing to such shortcomings, biochemical and molecular methods are increasingly being used to complement traditional methods in validating and/or providing information on dietary preferences of marine mammals (Iverson et al. 1997, Cherel et al. 2000, Deagle \& Tollit 2007). One such technique is fatty acid (FA) analysis. FA analysis is based on the principle that unique arrays of FAs from prey species can be transferred largely unaltered up the food chain where they are deposited in predator tissues that are high in lipid content (Iverson 1993). In marine mammals, milk is a source of dietary FA (Iverson 1993, Goebel 2002, Lea et al. 2002a, Littnan 2003). After the initial peri-natal fast, lactating fur seals regularly alternate between periods of foraging at sea and nursing their pups ashore. The rapid turnover rate of milk (production and consumption) implies that milk is derived from nutrients acquired while foraging (Iverson 1993, Iverson et al. 1997, Goebel 2002).

Milk FA integrates an individual's dietary information over a longer period of time when compared to scats and has been used qualitatively to infer spatial and temporal variation in diet and to identify specific prey (although recent studies indicate that using milk FA to infer prey-type consumed is not as straightforward as previously suggested) (Iverson et al. 1997, Brown et al. 1999, Goebel 2002, Lea et al. 2002a, Staniland \& Pond 2004, 2005).

The ability for FA to reflect differences in diet between ecological regions is dependant upon several factors, including: (1) the scale of spatial separation between regions and (2) prey communities within distinct ecological regions being measurably different in their FA profiles, with minimal overlap of species among regions (Bradshaw et al. 2003). The spatial separation between continental shelf and oceanic habitats utilised by New Zealand fur seals and the fact that they tend to travel directly to and from foraging grounds (Baylis et al. 2008a) suggest that the application of FA analysis to infer broad-scale dietary differences is well suited for this species.

Understanding and quantifying the type of foraging habitat used by New Zealand fur seals is important in further understanding their diet, in identifying critical habitats and in aiding management and conservation strategies. The present study aims at utilising conventional scat analysis and milk FA analysis to: (1) assess New Zealand fur seal diet using scats; (2) assess differences in FA composition of the milk of seals that for- 
aged on the continental shelf versus those that foraged in oceanic habitats; and (3) elucidate the spatial separation of foraging habitats within and between populations by predicting whether individuals foraged on the continental shelf or in oceanic habits based on their milk FA signatures.

\section{MATERIALS AND METHODS}

Study site. The present study was conducted at 4 colonies: (1) Cape Gantheaume ( $\left.36^{\circ} 04^{\prime} \mathrm{S}, 137^{\circ} 27^{\prime} \mathrm{E}\right)$ and (2) Cape du Couedic (36 $03^{\prime} \mathrm{S}, 136^{\circ} 42^{\prime} \mathrm{E}$ ) on Kangaroo Island; (3) North Neptune Island $\left(35^{\circ} 13^{\prime} \mathrm{S}\right.$, $\left.136^{\circ} 03^{\prime} \mathrm{E}\right) ;$ and (4) Liguanea Island (34 $59^{\prime} \mathrm{S}$, $135^{\circ} 37^{\prime} \mathrm{E}$ ) (Fig. 1). These colonies represent $\sim 79 \%$ of the Australian population of New Zealand fur seals Arctocephalus forsteri (Shaughnessy 2005).

Scat sampling and analysis. Scats from adult females were collected at random between March and October 2005 and April and September 2006 (Table 1). Scats were rinsed through 1.0 and $0.5 \mathrm{~mm}$ nested sieves to separate prey hard parts. Otoliths, eye lenses, feathers, scales and bones were stored dry, while cephalopod beaks were stored in $95 \%$ ethanol. Otoliths and cephalopod beaks were sorted and identified to species level where possible by comparison with our own South Australian Research and Development (SARDI) reference collection. Diet composition and the importance of prey were assessed by calculating the percent frequency of occurrence $(\mathrm{FO}$; proportion of samples containing a given prey species or group) and numerical abundance (NA; proportion of total number of individual prey).

Analysis of similarities (ANOSIM) using a Bray-Curtis dissimilarity matrix tested if FO or NA of prey consumed varied according to site or season (Primer Version 5.1.2, PRIMER-E). Where significant differences between main effects were found, differences were described using similarity of percentages analysis (SIMPER; Primer Version 5.1.2, PRIMER-E). Prey species occurring in
$<1 \%$ of scat samples, empty scats and scats that only contained unidentifiable prey remains (bones, scales, eye lenses) were excluded from analysis.

Separation of females as shelf or oceanic foragers. Data from the satellite-tracked females were obtained through the Argos satellite system. To further improve the accuracy of satellite tracks, the TimeTrack package (Version 1.0-9, M. D. Sumner, University of Tasmania), run through $\mathrm{R}$ statistical software, was used to apply the filter described by McConnell et al. (1992), based on a maximum horizontal speed of $2 \mathrm{~m} \mathrm{~s}^{-1}$ (Harcourt et al. 2002, Page et al. 2006).

Each foraging trip was classified as continental shelf or oceanic, based on the most distant foraging location. Because individuals that foraged in oceanic waters traversed the continental shelf, each satellite track was expressed as a percentage of the time spent in $5 \times 5 \mathrm{~km}$ grid cells, which enabled the percentage of total time spent on the continental shelf to be calculated for each seal. To determine the number of different $5 \times 5 \mathrm{~km}$ grid cells entered by each seal and the percentage of time they spent in each cell, we assumed a constant horizontal speed between the filtered locations and interpolated a new position for each hour along the satellite track using the timeTrack package (Page et al. 2006). The number of original and interpolated positions (located within $5 \times 5 \mathrm{~km}$ cells of a predetermined grid) were then summed and assigned to a central node. Values were converted to a percentage for each individual. Bathymetry was used to determine when a seal left continental shelf waters. Bathymetry values were calculated for each seal at 15 min intervals along each interpolated satellite track and were obtained from the General Bathymetric Chart of the Oceans (GEBCO) $1 \times 1 \mathrm{~km}$ World Bathymetry Grid (2.1).

Milk FA can originate from recent dietary intake and/or from mobilization of stored body fat, depending on when the seal last fed (Georges et al. 2001, Staniland \& Pond 2005, Budge et al. 2006). Milk FA of New Zealand fur seals on oceanic foraging trips may there-

Table 1. Arctocephalus forsteri. Sampling schedule which details the collection and number of milk and scat samples from lactating New Zealand fur seals at 4 breeding colonies in South Australia. -: no samples

\begin{tabular}{|c|c|c|c|c|c|c|c|c|}
\hline & \multicolumn{2}{|c|}{ Cape Gantheaume } & \multicolumn{2}{|c|}{ Cape du Couedic } & \multicolumn{2}{|c|}{ North Neptune Island } & \multicolumn{2}{|c|}{ Liguanea Island } \\
\hline & Milk & Scats & Milk & Scats & Milk & Scats & Milk & Scats \\
\hline \multicolumn{9}{|l|}{2005} \\
\hline Autumn & 15 & 180 & 4 & 113 & - & - & - & - \\
\hline Winter & 27 & 133 & 17 & 54 & - & - & - & - \\
\hline Spring & 19 & - & 3 & - & - & - & - & - \\
\hline \multicolumn{9}{|l|}{2006} \\
\hline Autumn & - & 51 & - & - & 9 & 45 & 11 & - \\
\hline Winter & 11 & - & 11 & - & 12 & 14 & - & - \\
\hline Spring & 11 & 94 & 10 & 35 & - & - & - & - \\
\hline Total & 83 & 458 & 45 & 202 & 21 & 59 & 11 & 0 \\
\hline
\end{tabular}



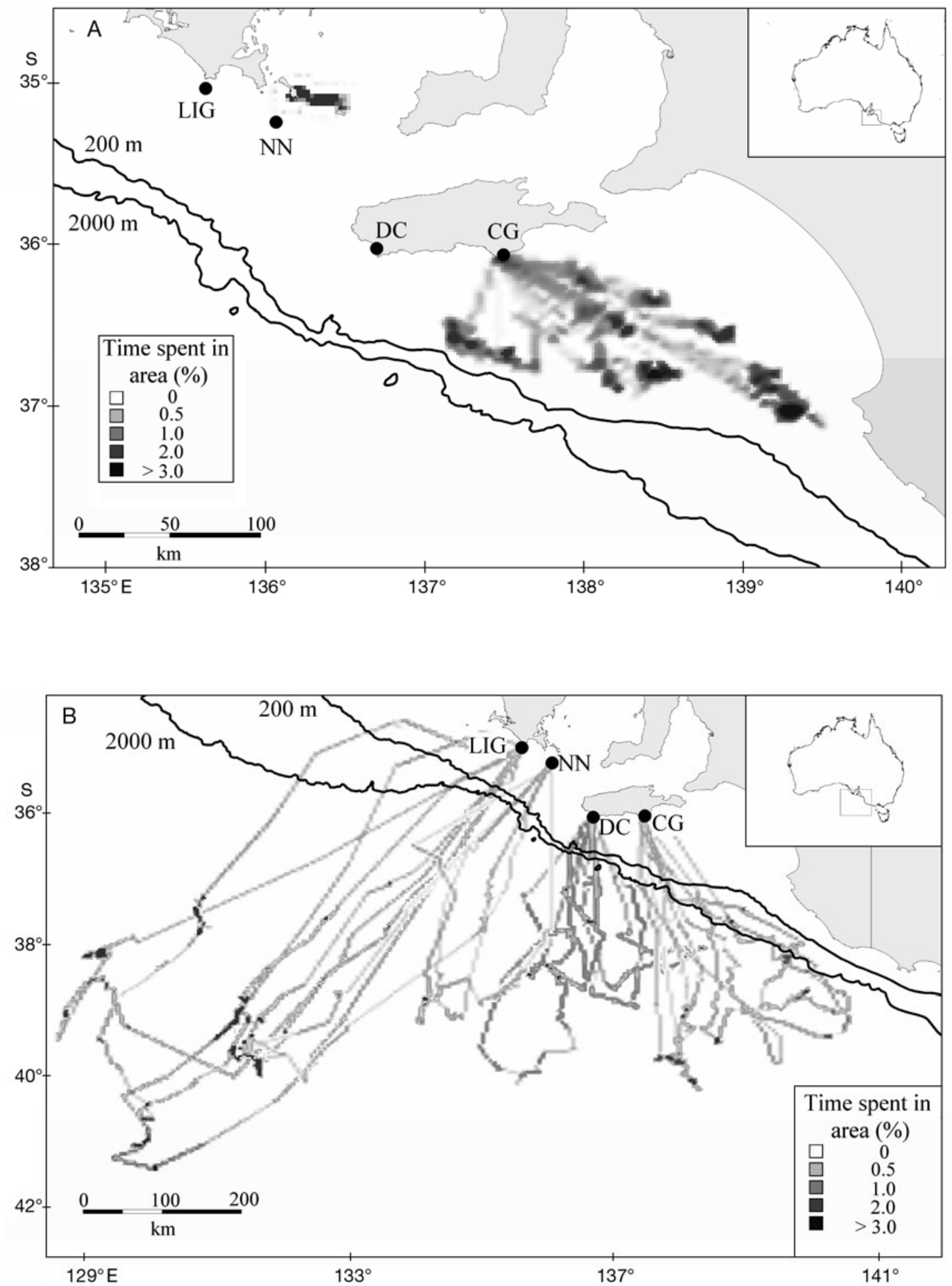

Fig. 1. Arctocephalus forsteri. The 4 colonies from which scat and milk samples were collected-LIG: Liguanea Island; NN: North Neptune Island; DC: Cape du Couedic; CG: Cape Gantheaume. Also presented are the foraging tracks from 29 lactating females satellite-tracked in 2005/2006 and the 200 and $2000 \mathrm{~m}$ depth contours. (A) Continental shelf foraging trips (CG $\mathrm{n}=10$ and $\mathrm{NN} n=1$ ). (B) Oceanic foraging trips (CG $\mathrm{n}=6, \mathrm{DC} \mathrm{n}=5$, NN $\mathrm{n}=3$ and LIG $\mathrm{n}=4$ ) 
fore reflect a combination of the most recent dietary intake and a longer dietary history. To ensure the foraging trip used to classify foraging location (i.e. the foraging trip that preceded milk sampling) was representative for each individual, females were tracked over multiple foraging trips.

Milk sampling. Milk samples were collected over a similar period as scat samples (Table 1). Milk samples were obtained from: (1) females fitted with satellite transmitters and (2) females selected at random (that were observed suckling a pup), but did not carry a satellite transmitter (Table 2). For females that carried a satellite tracker, milk samples were collected during removal of the unit, $<12 \mathrm{~h}$ after the seals return. Upon capture of satellite-tracked females, anaesthesia was induced and maintained using Isoflurane (Veterinary Companies of Australia) administered via a portable gas anaesthetic machine (Komesaroff Small Animal Anaesthetic Machine, Medical Developments Australia). Milk was manually expressed into $3 \times 2.5 \mathrm{ml}$ vials by massaging the teat after a $0.7 \mathrm{ml}$ intramuscular injection of Oxytocin (10 IU ml ${ }^{-1}$, Ilium Syntocin, Troy Laboratories). Milk samples collected from females fitted with satellite transmitters were classified as either continental shelf or oceanic based on foraging location. Milk samples from females selected at random were collected from non-sedated seals using the method described above. A small piece of fur was cut above the right fore flipper to identify females that had been randomly sampled. Milk from these females was categorized as random because their foraging locations were not known.

Milk FA analysis. Sample storage and analytical procedures were consistent with previous studies using pinniped milk FA (e.g. Lea et al. 2002a, Wheatley et al. 2008). Briefly, milk samples were frozen while in the field and stored at $-20^{\circ} \mathrm{C}$. In the laboratory, milk samples were thawed at room temperature and thoroughly mixed. A $50 \mathrm{mg}$ aliquot of milk was weighed, and lipid was quantitatively extracted overnight by a modified Bligh \& Dyer's (1959) 1-phase methanol/chloroform/water extraction (38 ml, 2:1:0.8, v/v/v). All solvents were of nanograde purity, thereby minimizing lipid oxidation. Phase separation was achieved the following day by the addition of chloroform and saline Milli Q water (1:1, v/v). The lower chloroform layer was removed, and total lipids were concentrated through rotary evaporation at $40^{\circ} \mathrm{C}$. Total lipids were transferred to glass vials, where they were reduced under a stream of nitrogen gas and made up to a known concentration by addition of chloroform. An aliquot was treated with methanol/concentrated hydrochloric acid/chloroform solution (10:1:1, v/v/v; $\left.3 \mathrm{ml}, 100^{\circ} \mathrm{C}, 60 \mathrm{~min}\right)$. After cooling and the addition of $1 \mathrm{ml}$ of water, FA methyl esters (FAME) were extracted into hexane/chloroform $(4: 1, \mathrm{v} / \mathrm{v} ; 3 \times 1.5 \mathrm{ml})$.

Gas chromatographic (GC) analyses of FAMEs were performed with an Agilent 7890A GC equipped with

Table 2. Arctocephalus forsteri. Number of milk samples collected at 4 breeding colonies from satellite-tracked lactating New Zealand fur seals (tracked) and lactating seals selected at random (random) and assigned to continental shelf or oceanic groups based on discriminant function analysis of milk fatty acid profiles. -: no samples

\begin{tabular}{|c|c|c|c|c|c|c|c|c|}
\hline & \multicolumn{2}{|c|}{ Cape Gantheaume } & \multicolumn{2}{|c|}{ Cape du Couedic } & \multicolumn{2}{|c|}{ North Neptune Island } & \multicolumn{2}{|c|}{ Liguanea Island } \\
\hline & Shelf & Oceanic & Shelf & Oceanic & Shelf & Oceanic & Shelf & Oceanic \\
\hline \multicolumn{9}{|l|}{ Autumn 2005} \\
\hline Tracked & 8 & 1 & - & - & - & - & - & - \\
\hline Random & 6 & - & 1 & 3 & - & - & - & - \\
\hline \multicolumn{9}{|l|}{ Winter 2005} \\
\hline Tracked & 2 & 3 & - & 2 & - & - & - & - \\
\hline Random & 12 & 10 & 1 & 14 & - & - & - & - \\
\hline \multicolumn{9}{|l|}{ Spring 2005} \\
\hline Tracked & - & 2 & - & - & - & - & - & - \\
\hline Random & 1 & 16 & - & 3 & - & - & - & - \\
\hline \multicolumn{9}{|l|}{ Autumn 2006} \\
\hline Tracked & - & - & - & - & 1 & - & - & 4 \\
\hline Random & - & - & - & - & 1 & 7 & - & 7 \\
\hline \multicolumn{9}{|l|}{ Winter 2006} \\
\hline Tracked & - & - & - & 2 & - & 3 & - & - \\
\hline Random & 4 & 7 & 2 & 7 & 3 & 6 & - & - \\
\hline \multicolumn{9}{|l|}{ Spring 2006} \\
\hline Tracked & - & - & - & 1 & - & - & - & - \\
\hline Random & 1 & 10 & 2 & 7 & - & - & - & - \\
\hline Combined total & 34 & 49 & 6 & 39 & 5 & 16 & 0 & 11 \\
\hline
\end{tabular}


a Supelco Equity-1 fused silica capillary column (15 m $\times 0.1 \mathrm{~mm}$ i.d., $0.1 \mu \mathrm{m}$ film thickness), a flame ionization detector $\left(290^{\circ} \mathrm{C}\right)$, a split/splitless injector $\left(290^{\circ} \mathrm{C}\right)$ and an Agilent 7683B auto-sampler. Helium was used as the carrier gas (425 kPa). After addition of an internal injection standard (19:0 FAME), samples were injected $(0.2 \mu \mathrm{l})$ in splitless mode at an oven temperature of $120^{\circ} \mathrm{C}$. After injection, the oven temperature was raised to $150^{\circ} \mathrm{C}$ at $10^{\circ} \mathrm{C} \mathrm{min}^{-1}$, then to $250^{\circ} \mathrm{C}$ at $17^{\circ} \mathrm{C} \mathrm{min}{ }^{-1}$ and finally to $270^{\circ} \mathrm{C}$ at $3^{\circ} \mathrm{C} \mathrm{min}{ }^{-1}$. A total of 54 ind. FAMEs were identified, with component identification by comparison of retention time data to those for authentic and laboratory standards (range 12:0 to 28:0) and using gas chromotography-mass spectrometer (GC-MS) data (Lea et al. 2002a) obtained using a Finnigan Thermoquest GCQ GC-MS fitted with an on-column injector and operated with Thermoquest Xcalibur software. The GC was fitted with a capillary column of polarity similar to that described above. Peaks were quantified with Agilent Technologies GC Chemstation software, with GC results subject to an error of $\pm 5 \%$ of individual component abundance.

Statistical analyses of milk samples. FA were presented as percentages of total FA and were arcsine transformed to improve normality (Zar 1996, Bradshaw et al. 2003). FA in greater than trace amounts (>0.5\%) were included in a reverse step-wise discriminant function analysis (DFA; SPSS 15.0). The DFA model was derived first from the satellite-tagged group of seals (the training set). DFA identified FA that were important in differentiating between seals that foraged on the continental shelf and those that foraged in distant oceanic waters. The resulting discriminant function was applied to the unknown 'random' individuals (the prediction set) to categorize them as to their foraging location based on milk FA analysis. Additional grouping variables were separately tested, and included: (1) colony (Cape Gantheaume, Cape du Couedic, North Neptune Island and Liguanea Island) and (2) season (autumn, winter and spring based on calendar month). Unless otherwise stated, all data are presented as means $\pm \mathrm{SD}$.

\section{RESULTS}

\section{Scats}

Prey remains were recovered from 719 scats of ArCtocephalus forsteri, 458 (64\%) of which were collected at Cape Gantheaume, 202 (28\%) collected from Cape du Couedic and 59 (8\%) from North Neptune Island. Thirty-three scats contained unidentifiable prey remains only and were excluded from analysis $(n=20$ from Cape Gantheaume, $\mathrm{n}=10$ from Cape du Couedic and $n=3$ from North Neptune Island). No scat samples were collected from Liguanea Island. Fish were the most common prey (78\%) with redbait Emmelichthys nitidus being the most common fish species, occurring in $26 \%$ of all scats $(\mathrm{n}=190)$. Myctophids Symbolophorus sp., Scopelosaurus sp. and Nansenia sp. were the only species representing oceanic regions (excluding the 'other seabird' group), and occurred in $7 \%$ of scats $(n=48)$. Gould's squid Nototodarus gouldi occurred in $19 \%$ of scats ( $\mathrm{n}=$ $139)$, and seabirds occurred in $4 \%$ of scats $(n=31)$. In general, redbait, Gould's squid and saury Scomberesox saurus were the most frequently occurring prey species at Cape Gantheaume (51, 20 and 11\% FO, respectively) (Table 3). At Cape du Couedic, redbait (49\% FO), Symbolophorus sp. (12\% FO), Gould's squid (15\% FO) and saury (9\% FO) were the most frequently occurring prey species, while at North Neptune Island, redbait ( $32 \%$ FO), Gould's squid (15\% FO), sweep Scorpis lineolatus (15\% FO) and jack mackerel Trachurus declivis (14\% FO) were the most frequently occurring prey species (Table 3).

Cape Gantheaume and Cape du Couedic were the only colonies in which scat samples were collected during the same season in the same year. As such, seasonal comparisons were limited to these colonies and for seasons when sample sizes allowed meaningful comparisons. Seasonal comparisons were made separately for each colony. There was no significant difference in the FO $(\mathrm{R}=0.004, \mathrm{p}=0.27)$ or NA $(\mathrm{R}=$ $0.012, p=0.13$ ) of prey items between autumn and winter 2005 at Cape Gantheaume or between autumn and winter 2005 at Cape du Couedic (FO: $\mathrm{R}=0.007$, $\mathrm{p}=0.32 ; \mathrm{NA}: \mathrm{R}=0.005, \mathrm{p}=0.29)$. Similarly, there were no significant differences in the FO $(R=-0.015$, $\mathrm{p}=0.73)$ or NA $(\mathrm{R}=-0.012, \mathrm{p}=0.60)$ of prey species between autumn and spring of 2006 at Cape Gantheaume.

No significant differences were evident in the FO (2-way crossed ANOSIM: $\mathrm{R}=0.017, \mathrm{p}=0.17$ ) or NA (2-way crossed ANOSIM: $\mathrm{R}=0.006, \mathrm{p}=0.29$ ) of prey species between Cape Gantheaume and Cape du Couedic in autumn and winter 2005 (Table 4). Conversely, there were significant differences between Cape Gantheaume and North Neptune Island in the FO of prey species in autumn $2006(\mathrm{R}=0.061, \mathrm{p}=$ 0.009) (Table 4). SIMPER analysis indicated redbait accounted for $26 \%$ of the difference between Cape Gantheaume and North Neptune Island, while Gould's squid and sweep accounted for 15 and $10 \%$, respectively. In autumn 2006, redbait and Gould's squid were more prevalent in scats from Cape Gantheaume, while sweep were more prevalent at North Neptune Island (Table 4). 
Table 3. Arctocephalus forsteri. Species list, percent numerical abundance (NA) and percent frequency of occurrence (FO) of the prey species identified in the scats of female New Zealand fur seals. Values in bold are the NA and FO for each prey type (fish, cephalopod and birds) and oceanic fish species. -: no samples

\begin{tabular}{|c|c|c|c|c|c|c|c|c|c|c|c|c|c|}
\hline \multirow{3}{*}{ Species } & \multirow{3}{*}{ Region } & \multicolumn{4}{|c|}{ Cape Gantheaume } & \multicolumn{4}{|c|}{ Cape du Couedic } & \multicolumn{4}{|c|}{ North Neptune Is. } \\
\hline & & \multicolumn{2}{|c|}{ NA } & \multicolumn{2}{|c|}{ FO } & & \multicolumn{2}{|c|}{$\mathrm{FO}$} & \multicolumn{2}{|c|}{ NA } & \multicolumn{2}{|c|}{$\mathrm{FO}$} \\
\hline & & $\mathrm{n}$ & $\%$ & $\mathrm{n}$ & $\%$ & $\mathrm{n}$ & $\%$ & $\mathrm{n}$ & $\%$ & $\mathrm{n}$ & $\%$ & $\mathrm{n}$ & $\%$ \\
\hline Fish & & 2468 & 90.3 & 395 & 86.2 & 872 & 88.8 & 185 & 91.6 & 157 & 80.9 & 48 & 81.4 \\
\hline Redbait (Emmelichthys nitidus) & Shelf/shelf-break & 1705 & 62.4 & 234 & 51.1 & 428 & 43.6 & 99 & 49.0 & 77 & 39.7 & 19 & 32.2 \\
\hline Saury (Scomberesox saurus) & Shelf/shelf-break & 275 & 10.1 & 54 & 11.8 & 51 & 5.2 & 19 & 9.4 & - & - & _- & - \\
\hline Anchovy (Engraulis australis) & Shelf/shelf-break & 80 & 2.9 & 25 & 5.5 & 7 & 0.7 & 5 & 2.5 & 1 & 0.5 & 1 & 1.7 \\
\hline Barracouta (Thyrsites atun) & Shelf/shelf-break & 68 & 2.5 & 28 & 6.1 & 10 & 1.0 & 7 & 3.5 & 6 & 3.1 & 3 & 5.1 \\
\hline $\begin{array}{l}\text { Slender bullseye (Parapriacanthus } \\
\text { elongatus) }\end{array}$ & Shelf/shelf-break & 52 & 1.9 & 24 & 5.2 & - & - & - & - & - & - & - & - \\
\hline Sardine (Sardinops sagax) & Shelf & 37 & 1.4 & 16 & 3.5 & 8 & 0.8 & 5 & 2.5 & 13 & 6.7 & 5 & 8.5 \\
\hline $\begin{array}{l}\text { Southern sea garfish (Hyporhamphus } \\
\text { melanochir) }\end{array}$ & Shelf/shelf-break & 21 & 0.8 & 6 & 1.3 & 1 & 0.1 & 1 & 0.5 & 1 & 0.5 & 1 & 1.7 \\
\hline Jack mackerel (Trachurus declivis) & Shelf/shelf-break & 19 & 0.7 & 8 & 1.7 & 29 & 3.0 & 8 & 4.0 & 12 & 6.2 & 8 & 13.6 \\
\hline Carangidae & Shelf/shelf-break & 13 & 0.5 & 8 & 1.7 & 2 & 0.2 & 2 & 1.0 & 1 & 0.5 & 1 & 1.7 \\
\hline Gemfish (Rexea solandri) & Shelf/shelf-break & 11 & 0.4 & 5 & 1.1 & - & - & - & - & 3 & 1.5 & 2 & 3.4 \\
\hline Flathead (Platycephalidae sp.) & Shelf/shelf-break & 10 & 0.4 & 4 & 0.9 & - & - & - & - & - & - & - & - \\
\hline Sweep (Scorpis lineolatus) & Shelf & 5 & 0.2 & 4 & 0.9 & 14 & 1.4 & 4 & 2.0 & 14 & 7.2 & 9 & 15.3 \\
\hline Gemphylidae & Shelf/shelf-break & 3 & 0.1 & 3 & 0.7 & 5 & 0.5 & 5 & 2.5 & - & - & - & - \\
\hline Red cod (Pseudophycis bacchus) & Shelf/shelf-break & 3 & 0.1 & 3 & 0.7 & - & - & - & - & - & - & - & - \\
\hline Australian salmon (Arripis trutta) & Shelf & 2 & 0.1 & 2 & 0.4 & - & - & - & - & 1 & 0.5 & 1 & 1.7 \\
\hline Silverbelly (Parequula melbournensis) & Shelf/shelf-break & 2 & 0.1 & 1 & 0.2 & - & - & - & - & - & - & - & - \\
\hline Leatherjacket (Monocanthidae sp.) & Shelf/shelf-break & 2 & 0.1 & 2 & 0.4 & 1 & 0.1 & 1 & 0.5 & - & - & - & - \\
\hline Tailor (Pomatomus saltatrix) & Shelf & 1 & 0.0 & 1 & 0.2 & - & - & - & - & 2 & 1.0 & 2 & 3.4 \\
\hline Apogonidae & Shelf-break & - & - & - & - & 4 & 0.4 & 2 & 1.0 & - & - & - & - \\
\hline Unidentified otolith & - & 61 & 2.2 & 42 & 9.2 & 31 & 3.2 & 25 & 12.4 & 6 & 3.1 & 5 & 8.5 \\
\hline Fish hard parts (vertebrae, scales, eye) & - & 8 & 0.3 & 8 & 1.7 & 5 & 0.5 & 5 & 2.5 & - & - & - & - \\
\hline Oceanic species & & 90 & 3.3 & 12 & 2.6 & 276 & 28.1 & 30 & 14.9 & 20 & 10.3 & 6 & 10.2 \\
\hline Myctophidae (Symbolophorus sp.) & Oceanic & 74 & 2.7 & 10 & 2.2 & 243 & 24.7 & 25 & 12.4 & 9 & 4.6 & 2 & 3.4 \\
\hline Scopelosaurus sp. & Oceanic & 14 & 0.5 & 5 & 1.1 & 11 & 1.1 & 7 & 3.5 & 1 & 0.5 & 1 & 1.7 \\
\hline Nansenia sp. & Oceanic & 2 & 0.1 & 2 & 0.4 & 22 & 2.2 & 6 & 3.0 & 10 & 5.2 & 4 & 6.8 \\
\hline Cephalopods & & 250 & 9.2 & 123 & 26.9 & 97 & 9.9 & 39 & 19.3 & 33 & 17.0 & 18 & 30.5 \\
\hline Gould's squid (Nototodarus gouldi) & Shelf & 220 & 8.1 & 94 & 20.5 & 87 & 8.9 & 30 & 14.9 & 28 & 14.4 & 13 & 15.1 \\
\hline Sepia sp. & Shelf & 2 & 0.1 & 1 & 0.2 & - & - & - & - & - & - & - & - \\
\hline Southern calamari (Sepioteuthis australis) & Shelf & 1 & - & 1 & 0.2 & - & - & - & - & - & - & - & - \\
\hline Unidenitified cephalopod & - & 8 & 0.3 & 8 & 1.7 & 4 & 0.4 & 3 & 1.5 & 1 & 0.5 & 1 & 1.2 \\
\hline Cephalopod hard parts (statolith, eye lens) & - & 19 & 0.7 & 19 & 4.1 & 6 & 0.6 & 6 & 3.0 & 4 & 2.1 & 4 & 4.7 \\
\hline Birds & & 14 & 0.5 & 14 & 3.1 & 13 & 1.3 & 13 & 6.4 & 4 & 2.1 & 4 & 4.7 \\
\hline Little penguin (Eudyptula minor) & Shelf & 10 & 0.4 & 10 & 2.2 & 9 & 0.9 & 9 & 4.5 & 4 & 2.1 & 4 & 4.7 \\
\hline Other seabird (Puffinus sp.) & Shelf or oceanic & 4 & 0.1 & 4 & 0.9 & 4 & 0.4 & 4 & 2.0 & - & - & - & - \\
\hline
\end{tabular}

\section{Satellite tracking}

Twenty-nine milk samples were collected from satellite-tracked females (18 in 2005 and 11 in 2006). Of the 29 females, 18 foraged off the continental shelf in oceanic waters and 11 foraged over the continental shelf (Fig. 1, Table 5). Females that foraged in oceanic waters spent the majority of their time in oceanic waters, with percentages of the trip time spent on the continental shelf ranging from 2.5 to $11.4 \%$ (Table 5). The mean distance and duration of foraging trips for females on the continental shelf was $134 \pm 36 \mathrm{~km}$ and $9.9 \pm 6.5 \mathrm{~d}$, respectively, while for oceanic waters the respective values were $525 \pm 215 \mathrm{~km}$ and $20.1 \pm 7.8 \mathrm{~d}$ (distance: Kruskal-Wallace ANOVA: $\chi^{2}=19.4$, df $=1$, $27, \mathrm{p}<0.001$; duration: ANOVA: $F_{1,27}=12.8, \mathrm{p}=0.001$ ) (Table 5).

\section{Milk}

A total of 160 milk samples were collected from New Zealand fur seals. Of these, 85 were collected in $2005-\mathrm{n}=61$ from Cape Gantheaume and $\mathrm{n}=24$ from Cape du Couedic. In 2006, 75 milk samples were collected $-\mathrm{n}=22$ from Cape Gantheaume, $\mathrm{n}=$ 


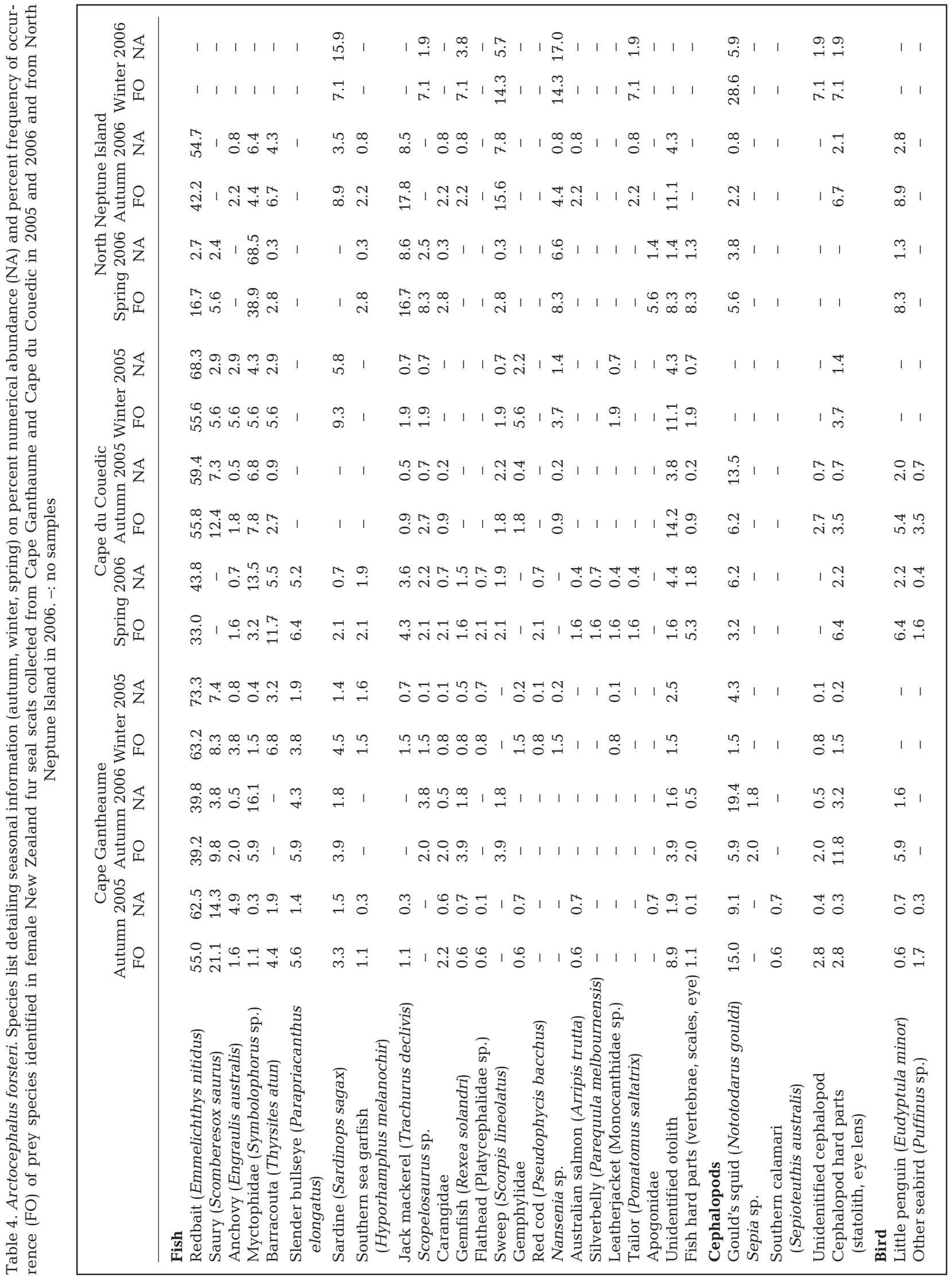


21 from Cape du Couedic, $\mathrm{n}=21$ from North Neptune Island and $\mathrm{n}=11$ from Liguanea Island (Tables $1 \& 2$ ). New Zealand fur seal milk was lipid rich containing a mean of $47 \pm 12 \%$ lipid content (wet mass). A total of 54 FA were routinely identified and quantified in fur seal milk lipid, with 18 FA found in greater than trace amounts (>0.5\%) and accounting for $95 \%$ of the total FA. The 6 most abundant FA (in decreasing percent abundance) were $18: 1 \mathrm{n}-9(25.4 \pm 4.6 \%)$, $16: 0(20.2 \pm 2.1 \%), 22: 6 \mathrm{n}-3(13.4 \pm 4.1 \%), 20: 1 \mathrm{n}-9+11$ $(7.5 \pm 6.4 \%), 16: 1 \mathrm{n}-7 \mathrm{c}(5.2 \pm 1.6 \%)$ and $14: 0(3.9 \pm$ $0.9 \%$ ) (Table 6$)$.

\section{FA composition of continental shelf versus oceanic waters}

Significant differences (Table 6) were recorded between satellite-tracked females from the continental shelf and oceanic groups in the proportion of saturated FA (SFA) $\left(F_{1,27}=69.1, \mathrm{p}<0.001\right)$, monounsaturated FA (MUFA) $\left(F_{1,27}=33.6, \mathrm{p}<0.001\right)$ and polyunsaturated FA (PUFA) $\left(F_{1,27}=18.3, \mathrm{p}<0.001\right)$. Seals foraging within oceanic waters were characterised by milk being comparatively high in MUFA $(47.4 \pm 4.4 \%)$ and lower in PUFA $(23.8 \pm 4.0 \%)$ when compared to seals

Table 5. Arctocephalus forsteri. Total length and girth data and characteristics of the foraging trip that preceded milk sampling recorded from 29 lactating New Zealand fur seals. Values are means \pm standard deviation

\begin{tabular}{|c|c|c|c|c|c|c|c|c|}
\hline $\begin{array}{l}\text { Location } \\
\text { Date of collection }\end{array}$ & ID & $\begin{array}{c}\text { Foraging } \\
\text { location }\end{array}$ & $\begin{array}{l}\text { No. of trips } \\
\text { prior to } \\
\text { milk sampling }\end{array}$ & $\begin{array}{l}\text { Total } \\
\text { length } \\
\text { (cm) }\end{array}$ & $\begin{array}{l}\text { Girth } \\
(\mathrm{cm})\end{array}$ & $\begin{array}{l}\text { Max. distance } \\
(\mathrm{km})\end{array}$ & $\begin{array}{c}\text { Trip duration } \\
\text { (d) }\end{array}$ & $\begin{array}{l}\text { Percent of trip } \\
\text { time spent on shel }\end{array}$ \\
\hline \multicolumn{9}{|l|}{ Cape Gantheaume } \\
\hline 26 Mar 2005 & 701 & Oceanic & 1 & 130 & 71 & 421 & 24.8 & 9.0 \\
\hline 29 Mar 2005 & 731 & Shelf & 3 & 124 & 63 & 144 & 11.7 & 100 \\
\hline 29 Mar 2005 & 691 & Shelf & 2 & 130 & 75 & 103 & 5.0 & 100 \\
\hline 3 Apr 2005 & 721 & Shelf & 4 & 138 & 73 & 82 & 7.7 & 100 \\
\hline 8 Apr 2005 & 711 & Shelf & 4 & 134 & 74 & 94 & 2.3 & 100 \\
\hline 7 May 2005 & 732 & Shelf & 3 & 137 & 75 & 107 & 17.2 & 100 \\
\hline 7 May 2005 & 712 & Shelf & 2 & 137 & 76 & 190 & 12.0 & 100 \\
\hline 8 May 2005 & 702 & Shelf & 5 & 130 & 71 & 126 & 3.3 & 100 \\
\hline 6 May 2005 & 692 & Shelf & 4 & 134 & 76 & 119 & 14.6 & 100 \\
\hline 7 Jul 2005 & 693 & Shelf & 5 & 138 & 74 & 128 & 11.3 & 100 \\
\hline 9 Jul 2005 & 474 & Oceanic & 3 & 131 & 67 & 329 & 7.3 & 11.4 \\
\hline 14 Jul 2005 & 733 & Oceanic & 3 & 143 & 74 & 548 & 22.7 & 8.6 \\
\hline 19 Aug 2005 & 763 & Oceanic & 3 & 131 & 68 & 326 & 14.0 & 10.2 \\
\hline 28 Aug 2005 & 723 & Shelf & 4 & 138 & 68 & 181 & 22.8 & 100 \\
\hline 6 Sep 2005 & 713 & Oceanic & 3 & 136 & 78 & 643 & 29.2 & 7.4 \\
\hline 1 Sep 2005 & 703 & Oceanic & 2 & 136 & 71 & 454 & 12.3 & 3.9 \\
\hline Mean & & & & $134 \pm 5$ & $72 \pm 4$ & $250 \pm 180$ & $13.6 \pm 8$ & $65.7 \pm 45.8$ \\
\hline \multicolumn{9}{|l|}{ Cape du Couedic } \\
\hline 13 Jun 2005 & 476 & Oceanic & 3 & 123 & 76 & 458 & 16.4 & 4.6 \\
\hline $22 \mathrm{Jul} 2005$ & 475 & Oceanic & 8 & 137 & - & 185 & 8.8 & 11.3 \\
\hline 7 Jul 2006 & 936 & Oceanic & 4 & 136 & 63 & 296 & 19.7 & 4.6 \\
\hline 13 Jul 2006 & 474 & Oceanic & 5 & 126 & 65 & 316 & 12.7 & 7.9 \\
\hline 2 Aug 2006 & 469 & Oceanic & 4 & 131 & 65 & 330 & 10.4 & 7.3 \\
\hline Mean & & & & $131 \pm 6$ & $67 \pm 6$ & $317 \pm 97$ & $13.6 \pm 4.4$ & $7.1 \pm 2.8$ \\
\hline \multicolumn{9}{|l|}{ Liguanea Island } \\
\hline 5 Apr 2006 & 472 & Oceanic & 1 & - & - & 798 & 23.0 & 5.1 \\
\hline 5 Apr 2006 & 960 & Oceanic & 1 & 135 & 79 & 802 & 26.4 & 4.8 \\
\hline 6 Apr 2006 & 468 & Oceanic & 1 & 126 & 74 & 811 & 32.4 & 5.0 \\
\hline 6 Apr 2006 & 961 & Oceanic & 1 & 126 & 65 & 881 & 24.6 & 2.5 \\
\hline Mean & & & & $129 \pm 5$ & $73 \pm 7$ & $831 \pm 39$ & $27.8 \pm 4$ & $4.4 \pm 1.2$ \\
\hline \multicolumn{9}{|c|}{ North Neptune Island } \\
\hline 8 Jun 2006 & 476 & Shelf & 19 & 126 & 74 & 63 & 1.4 & 100 \\
\hline 11 Jun 2006 & 475 & Oceanic & 2 & 135 & 79 & 658 & 24.7 & 3.9 \\
\hline 18 Jun 2006 & 473 & Oceanic & 3 & 129 & 76 & 728 & 31.0 & 4.6 \\
\hline 22 Jun 2006 & 953 & Oceanic & 3 & 131 & 76 & 475 & 21.0 & 10.8 \\
\hline Mean & & & & $132 \pm 4$ & $77 \pm 2$ & $620 \pm 298$ & $25.6 \pm 13$ & $29.8 \pm 46.9$ \\
\hline Mean shelf & & & & $133 \pm 5$ & $73 \pm 4$ & $134 \pm 36$ & $9.9 \pm 6.5$ & 100 \\
\hline Mean oceanic & & & & $132 \pm 5$ & $71 \pm 6$ & $525 \pm 215$ & $20.1 \pm 7.8$ & $6.8 \pm 2.9$ \\
\hline
\end{tabular}


Table 6. Arctocephalus forsteri. Fatty acid (FA) composition of New Zealand fur seal milk (\% of total FA). FA proportions ( $>0.5 \%$ ) are presented for satellite-tracked females that foraged within continental shelf waters and in oceanic waters and corresponding statistical significance between these 2 groups is also presented. FA proportions are also presented for the samples collected at random and assigned by discriminant function analysis to the continental shelf group and to the oceanic group. Values are means \pm SD. SFA: saturated fatty acids; MUFA: monounsaturated fatty acids; PUFA: polyunsaturated fatty acids; AA: arachidonic acid; EPA: eicosapentaenoic acid; DHA: docosahexaenoic acid

\begin{tabular}{|c|c|c|c|c|c|c|c|}
\hline \multirow[t]{2}{*}{ Fatty acid } & \multirow{2}{*}{$\begin{array}{l}\text { Total samples } \\
\quad(\mathrm{n}=160)\end{array}$} & \multicolumn{2}{|c|}{ Satellite-tracked animals } & \multirow[b]{2}{*}{$F_{1,27}$} & \multirow[b]{2}{*}{$\mathrm{p}$} & \multicolumn{2}{|c|}{ Randomly captured } \\
\hline & & $\begin{array}{l}\text { Continental shelf } \\
\quad(\mathrm{n}=11)\end{array}$ & $\begin{array}{c}\text { Oceanic waters } \\
\quad(\mathrm{n}=18)\end{array}$ & & & $\begin{array}{l}\text { Predicted oceanic } \\
\qquad(\mathrm{n}=34)\end{array}$ & $\begin{array}{l}\text { Predicted shelf } \\
\quad(\mathrm{n}=97)\end{array}$ \\
\hline Sum SFA & $29.7 \pm 2.4$ & $31.9 \pm 1.3$ & $28.7 \pm 0.8$ & 69.1 & $<0.001$ & $31.4 \pm 2.3$ & $28.9 \pm 2.1$ \\
\hline $14: 0$ & $3.8 \pm 0.9$ & $4.9 \pm 0.6$ & $3.6 \pm 0.4$ & 52.8 & $<0.000$ & $4.8 \pm 0.8$ & $3.5 \pm 0.7$ \\
\hline $15: 0$ & $0.9 \pm 0.2$ & $0.9 \pm 0.2$ & $1.0 \pm 0.2$ & 3.6 & 0.067 & $0.9 \pm 0.2$ & $0.9 \pm 0.2$ \\
\hline $16: 0$ & $20.2 \pm 2.1$ & $21.2 \pm 1.5$ & $19.0 \pm 0.9$ & 25.8 & $<0.000$ & $21.2 \pm 2.7$ & $19.9 \pm 2.0$ \\
\hline $17: 0$ & $0.7 \pm 0.1$ & $0.7 \pm 0.1$ & $0.7 \pm 0.1$ & 1.5 & 0.27 & $0.7 \pm 0.1$ & $0.7 \pm 0.1$ \\
\hline $18: 0$ & $2.9 \pm 0.6$ & $3.1 \pm 0.5$ & $3.0 \pm 0.5$ & 0.5 & 0.38 & $2.8 \pm 0.4$ & $2.8 \pm 0.6$ \\
\hline Sum MUFA & $45.2 \pm 7.9$ & $36.7 \pm 5.4$ & $47.4 \pm 4.4$ & 33.6 & $<0.001$ & $38.7 \pm 3.4$ & $48.1 \pm 7.6$ \\
\hline $16: 1 n-7$ & $5.2 \pm 1.7$ & $6.2 \pm 1.4$ & $4.7 \pm 1.4$ & 9.2 & 0.005 & $6.4 \pm 0.8$ & $4.9 \pm 1.8$ \\
\hline br17:1 & $0.5 \pm 0.2$ & $0.3 \pm 0.1$ & $0.6 \pm 0.2$ & 36.1 & $<0.000$ & $0.3 \pm 0.1$ & $0.5 \pm 0.2$ \\
\hline $17: 1 \mathrm{n}-8$ & $1.1 \pm 0.2$ & $0.9 \pm 0.1$ & $1.2 \pm 0.2$ & 25.2 & $<0.000$ & $1.0 \pm 0.1$ & $1.1 \pm 0.2$ \\
\hline $18: 1 n-7$ & $2.8 \pm 2.5$ & $2.9 \pm 0.5$ & $2.2 \pm 0.9$ & 5.3 & 0.028 & $3.0 \pm 0.6$ & $2.5 \pm 0.9$ \\
\hline $18: 1 n-9$ & $25.4 \pm 4.6$ & $22.4 \pm 4.9$ & $26.9 \pm 3.6$ & 9.1 & 0.005 & $22.5 \pm 3.6$ & $26.6 \pm 3.8$ \\
\hline $20: 1 n-9+11$ & $7.6 \pm 6.5$ & $2.2 \pm 1.3$ & $8.8 \pm 4.8$ & 30.2 & $<0.000$ & $2.8 \pm 1.3$ & $9.6 \pm 7.0$ \\
\hline $22: 1 n-9$ & $0.6 \pm 0.6$ & $0.2 \pm 0.1$ & $0.7 \pm 0.4$ & 37.3 & $<0.000$ & $0.2 \pm 0.1$ & $0.8 \pm 0.6$ \\
\hline Sum PUFA & $25.2 \pm 6.4$ & $31.4 \pm 5.5$ & $23.8 \pm 4.0$ & 18.3 & $<0.001$ & $30.0 \pm 4.0$ & $22.9 \pm 6.2$ \\
\hline $18: 2 n-6$ & $1.5 \pm 0.4$ & $2.1 \pm 0.4$ & $1.3 \pm 0.2$ & 54.3 & $<0.000$ & $1.9 \pm 0.3$ & $1.2 \pm 0.3$ \\
\hline $18: 4 n-3$ & $0.4 \pm 0.3$ & $1.1 \pm 0.3$ & $0.4 \pm 0.1$ & 52.8 & $<0.000$ & $0.6 \pm 0.2$ & $0.3 \pm 0.1$ \\
\hline $20: 4 n-3$ & $1.2 \pm 0.3$ & $1.6 \pm 0.2$ & $1.3 \pm 0.3$ & 4.4 & 0.049 & $1.4 \pm 0.2$ & $1.1 \pm 0.2$ \\
\hline $20: 4 n-6$ AA & $1.2 \pm 0.2$ & $1.1 \pm 0.2$ & $1.2 \pm 0.1$ & 0.3 & 0.53 & $1.3 \pm 0.3$ & $1.2 \pm 0.2$ \\
\hline 20:5n-3 EPA & $3.6 \pm 1.3$ & $5.5 \pm 0.9$ & $3.5 \pm 0.8$ & 35.7 & $<0.000$ & $4.6 \pm 0.9$ & $3.1 \pm 1.1$ \\
\hline $22: 5 n-3$ & $2.4 \pm 0.8$ & $3.0 \pm 0.5$ & $2.0 \pm 0.3$ & 45.9 & $<0.000$ & $3.1 \pm 0.5$ & $2.2 \pm 0.8$ \\
\hline $22: 6 n-3$ DHA & $13.4 \pm 4.1$ & $15.7 \pm 4.2$ & $12.9 \pm 2.8$ & 4.5 & 0.042 & $15.6 \pm 2.8$ & $12.4 \pm 4.3$ \\
\hline
\end{tabular}

that foraged in continental shelf waters (MUFA: $36.7 \pm$ 5.4; PUFA: $31.4 \pm 5.5 \%$ ). There were significant differences in the most abundant FA ( $\mathrm{p}<0.05$ in all cases), with 20:1n-9+11 and 18:1n-9 recording the greatest disparity between groups, both being recorded in higher proportions in seals foraging in oceanic waters (Table 6).

DFA identified 18:2n-6, 17:1n-8 and 18:1n-9 as adequate predictors of foraging location and provided the best discrimination among all models tested. Based on a standard and cross-validated classification matrices, overall correct assignment was $100 \%$ (Wilks' $\lambda=0.063$, approximate $\left.F_{1,27}=18.5, \mathrm{p}<0.0001\right)$. The FA 18:2n-6 accounted for $93 \%$ classification success, with 18:2n-6 and $17: 1 \mathrm{n}-8$ being the most important FA based on canonical scores. The resultant model that best discriminated females that foraged on the continental shelf from females that foraged in oceanic waters was: discriminant score $=-8.459+(0.281 \times 18: 1 \mathrm{n}-9)+$ $(2.851 \times 17: 1 \mathrm{n}-8)-(2.342 \times 18: 2 n-6)($ Fig. 2$)$.

Based on the above model, $74 \%(n=97)$ of milk samples collected at random were classified as oceanic, and $26 \%(n=34)$ were classified as continental shelf. Including satellite-tracked seals, this represented $59 \%$ (49 of 83 seals) classified as having foraged in oceanic waters from Cape Gantheaume, $87 \%$ from Cape du Couedic (39 of 45 seals), $76 \%$ from North Neptune Island (16 of 21 seals) and $100 \%(n=11)$ from Liguanea (Table 2). In total, $92 \%$ of the milk samples were assigned to the oceanic group, with $99 \%$ probability of correct assignment, while $74 \%$ of the milk samples collected at random were assigned to the continental shelf foraging group, with $99 \%$ probability of correct assignment (Table 7). The lower proportion of correctly assigned samples in the continental shelf group may reflect randomly sampled females within this group having foraged in both oceanic and continental shelf waters, or a previous foraging trip to oceanic waters. These results dramatically contrast with the scat analysis, which indicated that $2.6 \%(n=12)$ of scat samples from Cape Gantheaume contained oceanic prey; $14.9 \%(\mathrm{n}=30)$ at Cape du Couedic; and 10.2\% (n=6) at North Neptune Island (Table 3).

Cape Gantheaume was the only colony where seasonal variation in the predicted proportion of continental shelf foragers versus oceanic foragers was evident. In autumn $2005,7 \%$ of the milk samples ( $\mathrm{n}=1$ of 15 seals) were classified as oceanic. However, in winter 2005 and 2006, 48 and $64 \%$ of the milk samples ( $\mathrm{n}=13$ of 27 and 7 of 11 seals, respectively) were classified as 


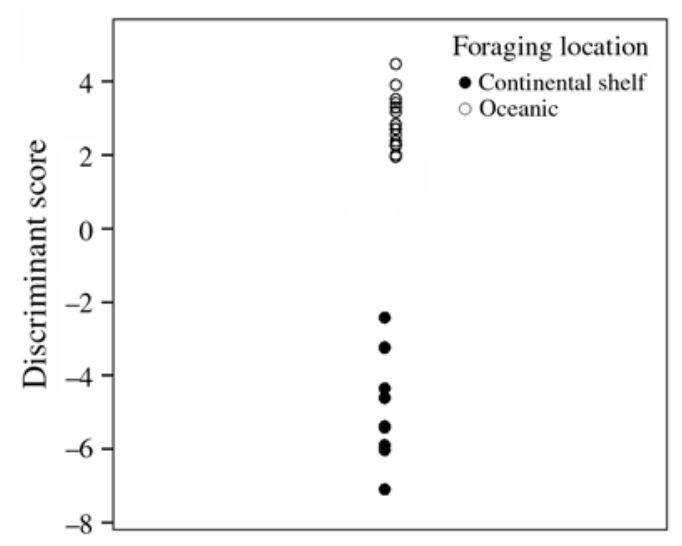

Fig. 2. Arctocephalus forsteri. Discriminant function analysis produced $100 \%$ correct assignment of satellite-tracked females (cross-validated) to their respective groups (continental shelf and oceanic) using the function: $-8.459+(0.281 \times$ $18: 1 \mathrm{n}-9)+(2.851 \times 17: 1 \mathrm{n}-8)-(2.342 \times 18: 2 \mathrm{n}-6)$

Table 7. Arctocephalus forsteri. Probability of correct grouping for 97 milk samples collected at random, which were assigned to the oceanic group, and 34 milk samples collected at random, which were assigned to the continental shelf group. Discriminant function scores were derived from milk samples collected from 29 satellite-tracked New Zealand fur seal females foraging in the 2 ecological zones (continental shelf [shelf] and oceanic waters [oceanic])

\begin{tabular}{|lcccc|}
\hline $\begin{array}{l}\text { Canonical } \\
\text { score }\end{array}$ & $\begin{array}{c}\text { Shelf } \\
(\mathrm{n}=34)\end{array}$ & $\begin{array}{c}\text { Mean } \\
\text { probability } \\
\text { shelf }\end{array}$ & $\begin{array}{c}\text { Oceanic } \\
(\mathrm{n}=97)\end{array}$ & $\begin{array}{c}\text { Mean } \\
\text { probability } \\
\text { oceanic }\end{array}$ \\
\hline$>4$ & 0 & - & 8 & 1 \\
3 & 0 & - & 29 & 1 \\
2 & 0 & - & 21 & 1 \\
1 & 0 & - & 12 & 1 \\
0 to 1 & 0 & - & 13 & 0.99 \\
-1 to 0 & 0 & - & 14 & 0.9 \\
-1 & 13 & 0.87 & 0 & - \\
-2 & 8 & 0.99 & 0 & - \\
-3 & 6 & 1 & 0 & - \\
$>-4$ & 7 & 1 & 0 & - \\
\hline
\end{tabular}

oceanic, and by spring 2005 and 2006, 95 and $91 \%$, respectively, of the milk samples collected were classified as oceanic ( $\mathrm{n}=18$ of 19 and 10 of 11 seals, respectively) $\chi^{-2}=26.4, \mathrm{df}=2, \mathrm{p}<0.001$ ).

\section{Spatial variation in FA composition}

To increase sample size, spatial variation was assessed using the combined satellite-tracked and randomly sampled data set. No meaningful statistical comparison could be made to test for spatial variation within the continental shelf group due to low sample sizes. For the oceanic group, winter 2006 was the only period in which multiple colonies (Cape Gantheaume, Cape du Couedic and North Neptune Island) were represented. Colony was a poor predictor of group membership. Despite poor classification success of only $52 \%$ ( $\mathrm{n}=13 / 25$, standard and cross-validated), differences in FA composition during winter were significant between colonies, suggesting dietary differences (Wilks' $\lambda=0.53$, approximate $F_{2,22}=14.8, \mathrm{p}<$ 0.001). In general, the greatest difference between colonies was in 20:1n-9+11, which was found in highest proportions at Cape du Couedic $(13 \pm 7.2 \%$ of FA proportion, range: 1.7 to $24.8 \%$ contribution of total FA content), when compared to Cape Gantheaume (7.2 \pm $6.3 \%)$, North Neptune Island (7.2 $\pm 3.2 \%)$ and Liguanea Island (8.4 $\pm 4.4 \%)$ (Kruskal-Wallace ANOVA, Chi-squared approximation, $\chi^{2}=20.1$, df $=3$, $\mathrm{p}<0.01)$.

\section{Seasonal variation in FA composition}

Seasonal variation in FA composition was assessed independently for each site using the combined satellite-tracked and randomly sampled data set. Cape Gantheaume was the only site at which there were sufficient replicates to permit analysis between seasons for the continental shelf group. A significant seasonal difference in 2005 between autumn and winter was evident, with DFA correctly classifying $82.1 \%$ of the samples (standard and cross-validated) to their original groups using the FA 16:0, 18:0 and 20:4n-3 (Wilks' $\lambda=$ 0.39, approximate $\left.F_{1,26}=12.5, \mathrm{p}<0.001\right)$. MUFAs where lower in autumn (35.8 \pm 4.1$)$ compared to winter $(39.4 \pm 4.0)\left(F_{1,26}=5.4, \mathrm{p}=0.03\right)$, with the MUFA $18: 1 \mathrm{n} 9$ recording the largest seasonal differences $\left(20.9 \pm 3.5\right.$ and $22.9 \pm 4.2$, respectively; $F_{1,26}=6.3, \mathrm{p}=$ 0.02). Significantly higher levels of 20:5n-3 were recorded in autumn compared to winter (5.5 \pm 1.0 and $4.5 \pm 0.9 ; F_{1,26}=13.5, \mathrm{p}=0.01$ ) (Table 8).

For the oceanic group, no seasonal variation in FA composition was evident between autumn and winter at North Neptune Island in 2006 (Wilks' $\lambda=0.17$, approximate $F_{1,14}=0.3, \mathrm{p}=0.8$ ) (Table 8). DFA discriminated between winter and spring at Cape Gantheaume in 2005 (80.6\% of samples correctly classified; Wilks' $\lambda=0.60$, approximate $F_{1,29}=19.1, \mathrm{p}<$ 0.001 ) on the basis of 20:5n-3. Similarly at Cape du Couedic, DFA discriminated between winter and spring in 2006 using 20:5n-3 with moderate classification success $(76.4 \%$ of samples correctly classified: Wilks' $\lambda=0.51$, approximate $F_{1,15}=14.3, \mathrm{p}=0.002$ ), suggesting seasonal differences in FA composition (Table 8). A pattern of decreasing PUFA and a concomitant increase in MUFA between winter and spring 
Table 8. Arctocephalus forsteri. Seasonal comparison of the FA composition of New Zealand fur seal milk (\% of total FA). FA proportions (>0.5\%) are presented for Cape Gantheaume 2005 for females foraging in continental shelf waters in autumn and winter and oceanic waters in winter and spring. Similarly seasonal comparisons are also presented for seals classified as oceanic foragers from Cape du Couedic in winter and spring and North Neptune Island between autumn and winter 2006. Values are means \pm SD. SFA: saturated fatty acids; MUFA: monounsaturated fatty acids; PUFA: polyunsaturated fatty acids; AA: arachidonic acid; EPA: eicosapentaenoic acid; DHA: docosahexaenoic acid

\begin{tabular}{|c|c|c|c|c|c|c|c|c|}
\hline \multirow[t]{2}{*}{ Fatty acid } & \multicolumn{2}{|c|}{$\begin{array}{l}\text { Cape Gantheaume } 2005 \\
\text { Continental shelf }\end{array}$} & \multicolumn{2}{|c|}{$\begin{array}{c}\text { Cape Gantheaume } 2005 \\
\text { Oceanic }\end{array}$} & \multicolumn{2}{|c|}{$\begin{array}{c}\text { Cape du Couedic } 2006 \\
\text { Oceanic }\end{array}$} & \multicolumn{2}{|c|}{$\begin{array}{c}\text { North Neptune Island } 2006 \\
\text { Oceanic }\end{array}$} \\
\hline & $\begin{array}{l}\text { Autumn } \\
(\mathrm{n}=14)\end{array}$ & $\begin{array}{l}\text { Winter } \\
(\mathrm{n}=14)\end{array}$ & $\begin{array}{l}\text { Winter } \\
(\mathrm{n}=13)\end{array}$ & $\begin{array}{l}\text { Spring } \\
(\mathrm{n}=18)\end{array}$ & $\begin{array}{l}\text { Winter } \\
(\mathrm{n}=9)\end{array}$ & $\begin{array}{l}\text { Spring } \\
(\mathrm{n}=8)\end{array}$ & $\begin{array}{c}\text { Autumn } \\
(\mathrm{n}=7)\end{array}$ & $\begin{array}{l}\text { Winter } \\
(\mathrm{n}=9)\end{array}$ \\
\hline Sum SFA & $32.4 \pm 2.4$ & $30.6 \pm 1.6$ & $29.1 \pm 1.4$ & $29.4 \pm 1.9$ & $28.6 \pm 1.8$ & $27.1 \pm 0.9$ & $29.9 \pm 3.1$ & $30.1 \pm 1.9$ \\
\hline $14: 0$ & $5.3 \pm 0.5$ & $5.0 \pm 0.7$ & $3.9 \pm 0.7$ & $3.6 \pm 0.6$ & $3.3 \pm 0.6$ & $2.7 \pm 0.2$ & $3.8 \pm 0.6$ & $3.8 \pm 0.3$ \\
\hline $15: 0$ & $1.0 \pm 0.2$ & $0.9 \pm 0.2$ & $0.9 \pm 0.2$ & $0.8 \pm 0.1$ & $0.8 \pm 0.1$ & $0.8 \pm 0.1$ & $1.0 \pm 0.1$ & $1.1 \pm 0.2$ \\
\hline $16: 0$ & $21.1 \pm 2.0$ & $20.5 \pm 2.1$ & $20.0 \pm 1.4$ & $20.5 \pm 2.3$ & $19.6 \pm 1.5$ & $18.5 \pm 1.3$ & $20.3 \pm 2.6$ & $19.7 \pm 1.9$ \\
\hline $17: 0$ & $0.8 \pm 0.1$ & $0.7 \pm 0.1$ & $0.7 \pm 0.1$ & $0.7 \pm 0.2$ & $0.7 \pm 0.1$ & $0.7 \pm 0.1$ & $0.7 \pm 0.1$ & $0.8 \pm 0.2$ \\
\hline $18: 0$ & $3.1 \pm 0.4$ & $2.6 \pm 0.4$ & $2.5 \pm 0.6$ & $2.6 \pm 0.8$ & $2.9 \pm 0.5$ & $3.1 \pm 0.8$ & $2.9 \pm 0.4$ & $3.1 \pm 0.2$ \\
\hline Sum MUFA & $35.8 \pm 4.1$ & $39.4 \pm 4.0$ & $42.8 \pm 5.5$ & $49.0 \pm 6.6$ & $48.0 \pm 5.4$ & $56.9 \pm 4.6$ & $43.8 \pm 8.3$ & $45.4 \pm 2.2$ \\
\hline $16: 1 n-7$ & $5.9 \pm 0.6$ & $6.7 \pm 0.8$ & $6.2 \pm 2.1$ & $5.6 \pm 2.5$ & $4.6 \pm 1.2$ & $3.4 \pm 1.0$ & $4.8 \pm 0.6$ & $4.7 \pm 1.1$ \\
\hline br17:1 & $0.3 \pm 0.1$ & $0.3 \pm 0.1$ & $0.5 \pm 0.1$ & $0.5 \pm 0.2$ & $0.5 \pm 0.1$ & $0.6 \pm 0.1$ & $0.5 \pm 0.2$ & $0.6 \pm 0.2$ \\
\hline $17: 1 \mathrm{n}-8$ & $0.9 \pm 0.1$ & $1.0 \pm 0.1$ & $1.2 \pm 0.2$ & $1.1 \pm 0.1$ & $1.1 \pm 0.1$ & $1.0 \pm 0.2$ & $1.1 \pm 0.1$ & $1.3 \pm 0.2$ \\
\hline $18: 1 n-7$ & $2.9 \pm 0.4$ & $3.3 \pm 0.4$ & $2.8 \pm 0.8$ & $3.0 \pm 0.9$ & $2.5 \pm 0.9$ & $1.8 \pm 0.6$ & $2.4 \pm 0.9$ & $2.0 \pm 1.0$ \\
\hline $18: 1 n-9$ & $20.9 \pm 3.5$ & $22.9 \pm 4.2$ & $24.4 \pm 2.2$ & $27.3 \pm 2.3$ & $26.4 \pm 3.0$ & $27.6 \pm 5.2$ & $26.7 \pm 6.2$ & $25.7 \pm 2.6$ \\
\hline $20: 1 n-9+11$ & $2.8 \pm 1.3$ & $3.0 \pm 1.5$ & $5.4 \pm 4.9$ & $8.7 \pm 6.6$ & $9.7 \pm 6.1$ & $18.6 \pm 5.0$ & $6.1 \pm 3.7$ & $8.0 \pm 2.6$ \\
\hline $22: 1 n-9$ & $0.2 \pm 0.1$ & $0.2 \pm 0.1$ & $0.4 \pm 0.4$ & $0.7 \pm 0.6$ & $0.9 \pm 0.5$ & $1.5 \pm 0.5$ & $0.5 \pm 0.3$ & $0.7 \pm 0.3$ \\
\hline Sum PUFA & $31.7 \pm 5.7$ & $29.9 \pm 4.2$ & $28.1 \pm 4.7$ & $21.6 \pm 5.4$ & $23.4 \pm 5.2$ & $16.0 \pm 4.0$ & $26.2 \pm 5.7$ & $24.5 \pm 3.7$ \\
\hline $18: 2 n-6$ & $2.1 \pm 0.2$ & $2.0 \pm 0.3$ & $1.4 \pm 0.3$ & $1.4 \pm 0.3$ & $1.2 \pm 0.3$ & $1.0 \pm 0.1$ & $1.3 \pm 0.2$ & $1.3 \pm 0.2$ \\
\hline $18: 4 n-3$ & $1.0 \pm 0.3$ & $0.7 \pm 0.3$ & $0.4 \pm 0.2$ & $0.3 \pm 0.1$ & $0.3 \pm 0.2$ & $0.1 \pm 0.0$ & $0.4 \pm 0.1$ & $0.4 \pm 0.1$ \\
\hline $20: 4 n-3$ & $1.6 \pm 0.2$ & $1.5 \pm 0.2$ & $1.2 \pm 0.3$ & $1.1 \pm 0.2$ & $1.1 \pm 0.2$ & $0.9 \pm 0.1$ & $1.3 \pm 0.3$ & $1.5 \pm 0.3$ \\
\hline $20: 4 n-6$ AA & $1.1 \pm 0.2$ & $1.3 \pm 0.2$ & $1.1 \pm 0.1$ & $1.2 \pm 0.2$ & $1.2 \pm 0.2$ & $1.0 \pm 0.1$ & $1.3 \pm 0.2$ & $1.3 \pm 0.2$ \\
\hline 20:5n-3 EPA & $5.5 \pm 1.0$ & $4.5 \pm 0.9$ & $4.0 \pm 0.8$ & $2.7 \pm 0.8$ & $3.3 \pm 0.7$ & $2.0 \pm 0.8$ & $3.7 \pm 1.0$ & $4.0 \pm 0.8$ \\
\hline $22: 5 n-3$ & $3.0 \pm 0.5$ & $3.1 \pm 0.5$ & $2.7 \pm 0.7$ & $2.2 \pm 0.7$ & $2.3 \pm 0.7$ & $1.4 \pm 0.3$ & $2.4 \pm 0.6$ & $2.3 \pm 0.7$ \\
\hline $22: 6 n-3$ DHA & $16.1 \pm 4.0$ & $15.5 \pm 2.9$ & $16.1 \pm 3.2$ & $11.4 \pm 3.7$ & $12.5 \pm 3.3$ & $8.1 \pm 2.7$ & $14.5 \pm 3.8$ & $12.3 \pm 4.8$ \\
\hline
\end{tabular}

was evident at Cape Gantheaume in 2005 and Cape du Couedic in 2006 (Table 8). The PUFA 22:6n-3 was found in higher proportions in winter compared to spring at Cape Gantheaume in $2005\left(F_{1,29}=6.3, \mathrm{p}=\right.$ 0.005) and Cape du Couedic in $2006\left(F_{1,15}=9.6, \mathrm{p}=\right.$ 0.007) (Table 8). The MUFA 20:1n-9+11 was found in significantly higher proportions in spring at Cape du Couedic only $\left(F_{1,15}=9.8, \mathrm{p}=0.007\right)$ (Table 8$)$. In general, samples with higher proportions of $20: 1 n-9+11$ had lower proportions of 22:6n-3 $\left(\mathrm{r}^{2}=-0.76, \mathrm{p}<0.001\right)$ and higher proportions of 22:1n-9 ( $\left.r^{2}=0.96, p<0.001\right)$.

\section{DISCUSSION}

Similar to studies on northern fur seals Callorhinus ursinus (Goebel 2002), FA analysis of New Zealand fur seal milk provided a reliable method to elucidate the spatial separation of foraging habitats within and between populations. With the use of milk FA, we were able to assess dietary information for particular individuals on a more appropriate spatio-temporal scale when compared to traditional methods. Results from FA analysis indicated that $74 \%$ of seals sampled were likely to have foraged in oceanic waters, while only $26 \%$ were likely to have foraged within continental shelf waters. These results were supported by concomitant satellite tracking, where $62 \%$ of satellitetracked seals foraged in oceanic waters. Cape Gantheaume was the only colony to record seasonal variation in the proportion of seals that foraged on the continental shelf. Based on FA analysis, seals from Cape Gantheaume shifted their foraging effort from continental shelf waters to oceanic waters from autumn to spring in 2005 and winter to spring in 2006. These results were also supported by a concomitant satellite tracking study at the Cape Gantheaume colony (Baylis et al. 2008b), which revealed that seasonal variation in foraging location was associated with seasonal changes in coastal upwelling activity.

FA results contrasted with scat analysis, where $7 \%$ of scats contained prey items associated with oceanic waters, suggesting only $7 \%$ of individuals sampled foraged in oceanic waters. This indicates that scats were biased toward females foraging on the continental shelf and/or the last foraging bout, if seals travelling from distant waters consumed prey on the continental shelf during the return trip. The discrepancy in results 
between scat analysis and FA analysis observed here supports previous studies that have highlighted the use of FA analysis as a powerful tool for assessing broad-scale spatial dietary patterns (Iverson et al. 1997, Goebel 2002, Bradshaw et al. 2003, Connan et al. 2005, 2007). The results also exemplify the difficulties encountered when quantifying the diet of wide-ranging marine predators using traditional methods and underscore the importance in understanding the limitations of the dietary method employed. In the case of New Zealand fur seals, results from scat analysis indicate that traditional methods are not representative of the diet of lactating females foraging in distant oceanic waters.

Our study highlights the importance of oceanic waters associated with the STF as critical foraging habitat for lactating New Zealand fur seals. The foraging trip distances and durations for lactating New Zealand fur seals travelling to distant ocean waters (see Baylis et al. 2008a) are among the longest reported for a temperate otariid species, with only lactating Subantarctic fur seals recording foraging trips of longer distance and duration (max. $1695 \mathrm{~km}$ and $54 \mathrm{~d}$ ) (Beauplet et al. 2004). While it is unclear whether these intrinsically long foraging trips are close to the limit of a central-place foraging fur seal, it does imply that lactating New Zealand fur seals may be vulnerable to climactic anomalies or anthropogenic impacts, such as climate change which will influence the location or productivity of the STF south of Australia, as projected for baleen whales that migrate to forage along Antarctic ocean fronts (Tynan \& Russell 2008).

Given the importance of the STF as foraging habitat for lactating New Zealand fur seals, additional information on prey species inhabiting waters associated with the STF south of Australia is required to further understand the diet of New Zealand fur seals and to assess their role as top predators in southern Australia. Additionally, without accounting for the fact that individuals within the same populations forage in discrete habitats, future dietary studies are unlikely to accurately reconstruct the diet of New Zealand fur seals in South Australia.

In contrast to the present study, traditional methods were able to reliably re-construct diet for Antarctic fur seals Arctocephalus gazella and northern fur seals Callorhinus ursinus (Zeppelin \& Ream 2006, Staniland et al. 2007). In these species, dietary differences were correlated with different ecological zones identified through satellite tracking (Staniland \& Boyd 2003, Robson et al. 2004). However, in Antarctic and northern fur seals, foraging trips to distant oceanic waters were, on average, $267 \pm 12 \mathrm{~km}$ and $8.2 \pm 0.8 \mathrm{~d}$ and $263 \pm 82 \mathrm{~km}$ and $8.8 \pm 2 \mathrm{~d}$, respectively (Staniland \& Boyd 2003, Robson et al. 2004). The mean oceanic for- aging trip distance for New Zealand fur seals was $525 \pm 215 \mathrm{~km}$, lasting $20.1 \pm 7.8 \mathrm{~d}$. Therefore, the greater distances that New Zealand fur seals travelled and the longer period of time spent in oceanic waters may explain why scat analysis was less successful in the current study and why FA results regarding foraging location and specific prey consumed were dramatically different from results derived from scat analysis.

Although scat analysis was unable to accurately reconstruct the diet of females that foraged in distant oceanic waters, it did provide fundamental information on species composition. The New Zealand fur seal is classified as a generalist predator, altering its diet over spatial and temporal scales. However, its diet tends to be dominated by only a small number of species at any one time (e.g. Lake 1997, Fea et al. 1999, Harcourt et al. 2002, Page et al. 2005). We found redbait Emmelichthys nitidus and Gould's squid Notodarus gouldi were the most frequently occurring prey species in scats. These species are likely to represent the primary diet of females foraging on the continental shelf. Redbait and squid are also important dietary components for lactating Australian fur seals Arctocephalus pusillus doriferus that foraged within continental shelf waters (Littnan et al. 2007), and have previously been recorded in the diet of New Zealand fur seals from Cape Gantheaume (Page et al. 2005).

Differences in the FO of prey species recovered from scats collected at Cape Gantheaume, Cape du Couedic and North Neptune Island also suggest that the species consumed within near-colony waters varies between colonies, and may reflect the proximity of each colony to the continental shelf-break. For example, Cape du Couedic is the closest colony to the continental shelfbreak ( 60 km accounting for colony-specific foraging direction) and recorded the greatest proportion of myctophids. Conversely, Cape Gantheaume and North Neptune are further from the continental shelf-break ( 110 and $\sim 80 \mathrm{~km}$, respectively), and continental shelf species dominated the prey species recovered from scats at these sites. Statistical analysis of inter-colony dietary differences was however limited by variability in the number of scats collected at each site and variability between colonies and between years in the season within which scat samples were collected.

The most abundant oceanic fish recovered from scat samples were myctophids Symbolophorus sp., which have been reported in other dietary studies of New Zealand fur seals in South Australia, Tasmania and New Zealand (Carey 1992, Lake 1997, Fea et al. 1999, Harcourt et al. 2002, Page et al. 2005). The high levels of 20:1n-9+11 MUFA recorded from the oceanic group may also suggest myctophids are important prey for females foraging in association with the STF, as 20:1n$9+11$ is relatively low in continental shelf species (e.g. 
redbait $4.2 \%$ and Gould's squid 4\%; A. Baylis \& P. Nichols unpubl. data), but is characteristically high in myctophids (e.g. Saito \& Murata 1998, Phleger et al. 1999, Lea et al. 2002b).

Seasonal variation in FA composition within the oceanic group was evident at Cape Gantheaume in 2005 and Cape du Couedic in 2006, where 22:6n-3 was recorded in higher proportions in winter than in spring. Although not apparent from scat analysis in our study, previous studies have identified seasonal variation in the occurrence of squid and fish recovered from the scats of New Zealand fur seals (Fea et al. 1999, Harcourt et al. 2002, Page et al. 2005). This is thought to primarily reflect changes in squid abundance, which is greater during summer and autumn, and declines in winter (Stark 2008). Whether seasonal differences in the proportions of selected FA reflected seasonal differences in the diet of seals or seasonal differences in the diet of their prey and, therefore, the FA composition of prey is unknown.

We did not attempt to relate milk FA to the FA ratios of potential prey because of: (1) the paucity of information relating to the FA profiles of New Zealand fur seal prey species and (2) the challenges associated with accounting for how particular dietary FAs are metabolised and deposited in predator tissue and in determining whether certain FA are selectively deposited or mobilized in the mammary gland (GrahlNielsen et al. 2003, Staniland \& Pond 2004, 2005, Budge et al. 2006).

\section{CONCLUSIONS}

Unlike other fur seal species, dietary information pertaining to New Zealand fur seals is limited. In South Australia, where $85 \%$ of the Australian population of New Zealand fur seals reside, dietary studies have identified the importance of continental shelf habitats for lactating females at the Cape Gantheaume colony (Goldsworthy et al. 2003, Page et al. 2005, 2006), the results of which have provided the basis for predictive models assessing the potential for trophic and operational fishery interaction across southern Australia (Goldsworthy et al. 2003, Goldsworthy \& Page 2007). With the aid of milk FA analysis and concomitant satellite tracking, we have also highlighted the importance of distant oceanic waters as foraging habitats for lactating New Zealand fur seals at 4 breeding colonies. Results indicated that oceanic waters were more important foraging habitats during autumn and winter than continental shelf waters. These results provide new information for understanding the habitat and management requirements of New Zealand fur seals. Traditional methods failed to account for the spatial separation of foraging habitats and were biased toward individuals foraging within continental shelf waters. FA analysis proved to be a valuable tool for assessing broad-scale spatial dietary patterns. Notwithstanding, for the FA approach to be further enhanced, the continued building of databases for prey items is required, including examining seasonal and regional differences for key species. Steps toward further understanding the trophic relationships between New Zealand fur seals and their prey will be dependant on additional information pertaining to the distribution and occurrence of fish and cephalopod species inhabiting waters associated with the STF south of Australia and the continued development and application of alternative dietary techniques, used in combination with traditional dietary analysis.

Acknowledgements. The present study was supported through Nature Foundation SA, MA Ingram Trust, Holsworth Wildlife fund, Project AWARE, Wildlife Conservation Fund, Sea World Research and Rescue Foundation, Rossi Boots Australia and the Fisheries Research and Development Corporation (FRDC) Grants Scheme (PN 2005/031). A.M.M.B received an Australian Postgraduate Award to conduct this research. We thank T. Ward (SARDI) for securing FRDC funding, and S. Goldsworthy, D. Paton (Adelaide University) and T. Ward for ongoing support of the project. M. Miller, B. Mooney, M. Guest and P. Mansour (CSIRO Marine and Atmospheric Research) provided guidance and advice with sample preparation. We thank the many volunteers who assisted with fieldwork, in particular, D. Lierch and C. 'Dutchie' Pleuis. For support in Hobart we thank J. StuartSmith, R. Stuart-Smith, P. Lewis and R. Cook. This manuscript was improved by helpful comments from B. Page and 4 anonymous reviewers. Research was conducted under the Adelaide University animal ethics permit S80-2004 and the Department for Environment and Heritage permit A24684-3.

\section{LITERATURE CITED}

Baylis AMM, Page B, Goldsworthy SD (2008a) Colony specific foraging areas of lactating New Zealand fur seals. Mar Ecol Prog Ser 361:279-290

> Baylis AMM, Page B, Goldsworthy SD (2008b) Effect of seasonal changes in upwelling activity on the foraging locations of a wide-ranging central place forager, the New Zealand fur seal. Can J Zool 86:774-789

Baylis AMM, Hamer DJ, Nichols PD (2009) Assessing the use of milk fatty acids to infer the diet of the Australian sea lion (Neophoca cinerea). Wildl Res 36:169-176

> Beauplet G, Dubroca L, Guinet C, Cherel Y, Dabin W, Gagne C, Hindell M (2004) Foraging ecology of subantarctic fur seals Arctocephalus tropicalis breeding on Amsterdam Island: seasonal changes in relation to maternal characteristics and pup growth. Mar Ecol Prog Ser 273:211-225

> Bligh EG, Dyer WJ (1959) A rapid method of total lipid extraction and purification. Can J Biochem Physiol 37:911-917

$>$ Bodley KB, Mercer JR, Bryden MM (1999) Rate of passage of digesta through the alimentary tract of the New Zealand fur seal (Arctocephalus forsteri) and the Australian sea lion (Neophoca cinerea). Aust J Zool 47:193-198 
Bradshaw CJA, Hindell MA, Best NJ, Phillips KL, Wilson G, Nichols PD (2003) You are what you eat: describing the foraging ecology of southern elephant seals (Mirounga leonina) using blubber fatty acids. Proc R Soc Lond B Biol Sci 270:1283-1292

Brown DJ, Boyd IL, Cripps GC, Butler PJ (1999) Fatty acid signature analysis from the milk of Antarctic fur seals and southern elephant seals from South Georgia: implications for diet determination. Mar Ecol Prog Ser 187:251-263

Budge SM, Iverson SJ, Koopman HN (2006) Studying trophic ecology in marine ecosystems using fatty acids: a primer on analysis and interpretation. Mar Mamm Sci 22:759-801

Carey PW (1992) Fish prey species of the New Zealand fur seal (Arctocephalus forsteri). N Z J Ecol 16:41-46

- Chaurand T, Weimerskirch H (1994) The regular alternation of short and long foraging trips in the blue petrel Halobaena caerulea: a previously undescribed strategy of food provisioning in a pelagic seabird. J Anim Ecol 63:275-282

> Cherel Y, Hobson KA, Weimerskirch H (2000) Using stableisotope analysis of feathers to distinguish moulting and breeding origins of seabirds. Oecologia 122:155-162

- Connan M, Mayzaud P, Boutoute M, Weimerskirch $\mathrm{H}$, Cherel Y (2005) Lipid composition of stomach oil in a procellariiform seabird Puffinus tenuirostris: implications for food web studies. Mar Ecol Prog Ser 290:277-290

Connan M, Cherel Y, Mabille G, Mayzaud P (2007) Trophic relationships of white-chinned petrels from Crozet Islands: combined stomach oil and conventional dietary analyses. Mar Biol 152:95-107

> Deagle BE, Tollit DJ (2007) Quantitative analysis of prey DNA in pinniped faeces: Potential to estimate diet composition? Conserv Genet 8:743-747

Dellinger T, Trillmich F (1988) Estimating diet composition from scat analysis in otariid seals (Otariidae): Is it reliable? Can J Zool 66:1865-1870

Fea NI, Harcourt R, Lalas C (1999) Seasonal variation in the diet of New Zealand fur seals (Arctocephalus forsteri) at Otago Peninsula, New Zealand. Wildl Res 26:147-160

> Georges JY, Groscolas R, Guinet C, Robin JP (2001) Milking strategy in subantarctic fur seals Arctocephalus tropicalis breeding on Amsterdam Island: evidence from changes in milk composition. Physiol Biochem Zool 74:548-599

Goebel ME (2002) Northern fur seal lactation, attendance and reproductive success in two years of contrasting oceanography. PhD thesis, University of California, Santa Cruz, CA

Goldsworthy SD, Page B (2007) A risk-assessment approach to evaluating the significance of seal bycatch in two Australian fisheries. Biol Conserv 139:269-285

Goldsworthy SD, Bulman C, He X, Larcombe J, Littnan C (2003) Trophic interactions between marine mammals and Australian fisheries: an ecosystem approach. In: Gales N, Hindell M, Kirkwood R (eds) Marine mammals and humans: fisheries, tourism and management. CSIRO Publications, Hobart, p 62-99

> Grahl-Nielsen O, Anderson M, Derocher AE, Lyderson C, Wiig O, Kovacs KM (2003) Fatty acid composition of the adipose tissue of polar bears and their prey: ringed seals, bearded seals and harp seals. Mar Ecol Prog Ser 265: 275-282

Gremillet D, Dell'Omo G, Ryan PG, Peters G, Ropert-Coudert Y, Weeks SJ (2004) Offshore diplomacy, or how seabirds mitigate intra-specific competition: a case study based on GPS tracking of cape gannets from neighboring colonies. Mar Ecol Prog Ser 268:265-279

Harcourt RG, Bradshaw CJA, Dickson K, Davis LS (2002) Foraging ecology of a generalist predator, the New Zealand fur seal. Mar Ecol Prog Ser 227:11-24
Iverson SJ (1993) Milk secretion in marine mammals in relation to foraging: Can milk fatty acids predict diet? Symp Zool Soc Lond 66:263-291

Iverson SJ, Arnould JPY, Boyd IL (1997) Milk fatty acid signatures indicate both major and minor shifts in the diet of lactating Antarctic fur seals. Can J Zool 75:188-197

Lake S (1997) Analysis of the diet of New Zealand fur seals Arctocephalus forsteri in Tasmania. In: Hindell MA, Kemper C (eds) Marine mammal research in the southern hemisphere, Vol 1 - status, ecology and medicine. Surrey Beatty, Sydney, p 125-129

Lea MA, Cherel Y, Guinet C, Nichols PD (2002a) Antarctic fur seals foraging in the Polar Frontal Zone: inter-annual shifts in diet as shown from faecal and fatty acid analysis. Mar Ecol Prog Ser 245:281-297

Lea MA, Nichols PD, Wilson G (2002b) Fatty acid composition of lipid-rich myctophids and mackerel icefish (Champsocephalus gunnari) - Southern Ocean food-web implications. Polar Biol 25:843-854

Littnan CL (2003) Approaches to studying the foraging ecology of the Australian fur seal Arctocephalus pusillus doriferus in northern Bass Strait. PhD thesis, Macquarie University, Sydney

Littnan CL, Arnould JPY, Harcourt RG (2007) Effect of proximity to the shelf edge on the diet of female Australian fur seals. Mar Ecol Prog Ser 338:257-267

McConnell BJ, Chambers C, Fedak MA (1992) Foraging ecology of southern elephant seals in relation to the bathymetry and productivity of the Southern Ocean. Antarct Sci 4:393-398

Page B, McKenzie J, Goldsworthy SD (2005) Dietary resource partitioning among sympatric New Zealand and Australian fur seals. Mar Ecol Prog Ser 293:283-302

Page B, McKenzie J, Sumner MD, Coyne M, Goldsworthy SD (2006) Spatial separation of foraging habitats among New Zealand fur seals. Mar Ecol Prog Ser 323:263-279

Paine RT (1988) Food webs: road maps of interactions or grist for theoretical development? Ecology 69:1648-1654

Phleger CF, Nelson MW, Mooney BD, Nichols PD (1999) Wax ester versus triacylglycerols in myctophid fishes from the Southern Ocean. Antarct Sci 11:436-444

Raclot T, Groscolas R, Cherel Y (1998) Fatty acid evidence for the importance of myctophid fishes in the diet of king penguins, Aptenodytes patagonicus. Mar Biol 132: $523-533$

Robson BW, Goebel ME, Baker JD, Ream RR and others (2004) Separation of foraging habitat among breeding sites of a colonial marine predator, the northern fur seal (Callorhinus ursinus). Can J Zool 82:20-29

Saito H, Murata M (1998) Origin of the monoene fats in the lipid of midwater fishes: relationship between the lipids of myctophids and those of their prey. Mar Ecol Prog Ser 168:21-33

Shaughnessy PD (2005) Population assessment of New Zealand fur seals and Australian sea lions at some colonies in South Australia, 2004-05. CSIRO Sustainable Ecosystems, Canberra

Staniland IJ (2002) Investigating the biases in the use of hard prey remains to identify diet composition using Antarctic fur seals (Arctocephalus gazella) in captive feeding trials. Mar Mamm Sci 18:223-243

Staniland IJ, Boyd IL (2003) Variation in the foraging location of Antarctic fur seals (Arctocephalus gazella) and the effects on diving behaviour. Mar Mamm Sci 19:331-343

Staniland IJ, Pond D (2004) Variability in milk fatty acids: recreating a foraging trip to test dietary predictions in Antarctic fur seals. Can J Zool 82:1099-1107 
Staniland IJ, Pond DW (2005) Investigating the use of milk fatty acids to detect dietary changes: a comparison with faecal analysis in Antarctic fur seals. Mar Ecol Prog Ser 294:283-294

Staniland IJ, Boyd IL, Reid K (2007) An energy-distance trade-off in a central-place forager, the Antarctic fur seal (Arctocephalus gazella). Mar Biol 152:233-241

Stark KE (2008) Ecology of the arrow squid (Nototodarus gouldi) in southeastern Australian waters: a multi-scale investigation of spatial and temporal variability. $\mathrm{PhD}$ thesis, University of Tasmania, Hobart

Tynan CT, Russell JL (2008) Assessing the impacts of future 2

Editorial responsibility: Hans Heinrich Janssen,

Oldendorf/Luhe, Germany degree global warming on South Ocean cetaceans. In: Document SC/60/E3 WWF, 2007, Climate solutions, WWF's vision for 2050

- Wheatley KE, Nichols PD, Hindell MA, Harcourt RG, Bradshaw CJA (2008) Differential mobilization of blubber fatty acids in lactating Weddell seals: evidence for selective use. Physiol Biochem Zool 81:651-662

Zar JH (1996) Biostatistical analysis. Prentice-Hall, Upper Saddle River, NJ

Zeppelin TK, Ream RR (2006) Foraging habitats based on the diet of female northern fur seals (Callorhinus ursinus) on the Pribilof Islands, Alaska. J Zool (Lond) 270:565-576

Submitted: August 21, 2008; Accepted: January 6, 2009 Proofs received from author(s): March 27, 2009 